\title{
Slow dynamics via degenerate variational asymptotics
}

\author{
By Georg A. Gottwald ${ }^{1}$ and Marcel Oliver ${ }^{2}$ \\ ${ }^{1}$ School of Mathematics and Statistics, University of Sydney, NSW 2006, \\ Australia, georg.gottwald@sydney.edu.au \\ ${ }^{2}$ School of Engineering and Science, Jacobs University, 28759 Bremen, Germany, \\ oliver@member.ams.org
}

We introduce the method of degenerate variational asymptotics for a class of singularly perturbed ordinary differential equations in the limit of strong gyroscopic forces. Such systems exhibit dynamics on two separate time scales. We derive approximate equations for the slow motion to arbitrary order through an asymptotic expansion of the Lagrangian in suitably transformed coordinates. We prove that the necessary near-identity change of variables can always be constructed, and that solutions of the slow limit equations shadow solutions of the full parent model at the expected order over a finite interval of time.

Keywords: Multiple scale methods, Lagrangian mechanics, gyroscopic forces, perturbation theory, slow manifolds, geometric mechanics

\section{Introduction}

We consider systems of differential equations of the form

$$
\begin{aligned}
\dot{q} & =p, \\
\varepsilon \dot{p} & =J p-\nabla V(q),
\end{aligned}
$$

where $q:[0, T] \rightarrow \mathbb{R}^{2 d}$ is the vector of positions, $p$ the vector of corresponding momenta, $J$ is the canonical symplectic matrix in $2 d$ dimensions, $V$ is a smooth potential and $\varepsilon$ is a small parameter. The system supports slow motion on the time scale $t=O(1)$ and fast motion on the time scale $t=O\left(\varepsilon^{-1}\right)$. Our goal is to study variational approximations of the motion on a quasi-invariant slow manifold.

In the simplest physical interpretation of (1.1) with $d=1$, the model describes the motion of a single charged particle in a planar potential $V$ under the influence of a magnetic field normal to the plane of motion. The limit $\varepsilon \rightarrow 0$ corresponds to the somewhat unphysical situation that the mass of the particle is going to zero while its charge is held constant.

The motivation for studying (1.1) as written comes from the observation that the model arises in direct analogy to the semigeostrophic limit in geophysical fluid dynamics (Cotter \& Reich 2006; Oliver 2006; Gottwald et al. 2007; Frank \& Gottwald 2013). (This limit is also known as "Phillips type 2 scaling" or the "frontal dynamics regime.") In this interpretation, $J \dot{q}$ represents the Coriolis force and the limit $\varepsilon \rightarrow 0$ is the limit of a rapidly rotating earth. For the geophysical background and extended bibliography, we refer the reader to Salmon (1998), Cullen (2006) and Vanneste (2013). 
System (1.1) is Hamiltonian, albeit with a noncanonical symplectic structure. Hence, we would expect that there exist approximate slow manifolds in the sense that they are almost invariant under the evolution and on which the dynamics is given by an equation of the form $\dot{q}=F_{\text {slow }}(q)$ which can be constructed as an asymptotic series in $\varepsilon$ up to a small remainder (MacKay 2004).

We note that the equations of motion for the slow degrees of freedom cannot be determined by direct inspection, except for the leading order term obtained by setting $\varepsilon=0$ in (1.1). However, there are several ways to construct $F_{\text {slow }}(q)$ to any order. Most directly, we can re-insert the slow equation at order $\varepsilon^{n}$ to eliminate $\dot{p}$ at order $\varepsilon^{n+1}$, iterating up order by order. The construction can be made rigorous using an iteratively constructed sequence of transformations that separate fast and slow motions, which we shall review in Section 2. These transformations generally neither rely on nor preserve the Hamiltonian structure and would work equally well if $\nabla V$ were replaced by an arbitrary vector field. It is possible, though, to choose a canonical transformation in the Hamiltonian case as pointed out for example, by Neusshtadt (1984) in the context of averaging. We note that classical averaging also yields an equation for the amplitudes of the fast motions which, in general, can resonate with the slow dynamics. Here, however, we are only concerned with the dynamics on slow manifolds where resonances are not a concern. For a more recent account on slow-fast splittings in a variety of related contexts, see Cheng \& Mahalov (2013) and references therein.

A second approach in the Hamiltonian context is the use of normal form transformations (Gelfreich \& Lerman 2002; Murdock 2003; Sanders et al. 2007; Uldall Kristiansen \& Wulff 2012). This technique has been applied by Cotter and Reich (2006) to a system of precisely the form (1.1) showing, in particular, that it is possible to construct an exponentially accurate slow manifold.

In this paper, we analyze a new construction which is based on the variational formulation of (1.1). Namely, the system is equivalent to the Euler-Lagrange equation for the Lagrangian

$$
L_{\varepsilon}(q, \dot{q})=\frac{\varepsilon}{2}|\dot{q}|^{2}-\frac{1}{2} \dot{q}^{T} J q-V(q),
$$

which reads

$$
\varepsilon \ddot{q}-J \dot{q}+\nabla V(q)=0 .
$$

Our approach is based on the observation that, formally, the leading order Lagrangian is affine, i.e. is linear in the velocity. We shall construct our approximate slow vector field by applying carefully chosen near-identity transformations to the Lagrangian that push the non-affine contributions into terms which are of successively increasing order in $\varepsilon$. Then, by truncating the $\varepsilon$-expansion of the Lagrangian, we drop all non-affine terms. As we will show in this paper, these equations provide an approximation to the slow dynamics accurate to the order to which the procedure is carried out.

We call this approach the method of degenerate variational asymptotics. The term variational asymptotics generally refers to perturbation techniques that are performed at the level of the variational principle. We say it is degenerate because the affine target Lagrangian is degenerate, in fact totally singular (Popescu 2009), which implies that the phase space is constrained to $\mathbb{R}^{2 d}$, the expected dimension of the slow manifold. 
The construction is based on ideas first developed in the context of rotating fluid flow (Oliver 2006). It has three distinct advantages. First, the resulting slow equations are again variational, therefore, under suitable restriction of phase space, also Hamiltonian (Oliver \& Vasylkevych 2011). Second, the new construction involves only differentiation and is therefore easy to carry out in an automated way. Third, the transformation which renders the Lagrangian affine to some given order is by far not unique. For systems of ordinary differential equations of the form (1.1), all of the possible resulting slow systems are asymptotically equivalent as we shall show below. For partial differential equations, however, the choices that can be made will affect the functional setting, hence the well-posedness of the slow system as an initial value problem (Oliver \& Vasylkevych 2013, 2014). Based on these examples, we expect that the freedom in the choice of transformation will be crucial to proving similarly strong results in the PDE context.

One of the crucial observations in the PDE context, more specifically for semigeostrophic theory, is that a transformation to an (essentially) canonical Hamiltonian slow system as originally suggested by Salmon (1985) turns out to be precisely the situation when well-posedness of the Cauchy problem breaks down. This has led us to consider alternative constructions of the type considered here which allow us to manipulate both, the symplectic structure and the Hamiltonian, in a controlled way. Hence, we see the present work as a first step towards establishing corresponding results for infinite dimensional reduced slow dynamics.

Our main result is of shadowing type: when the solution to the full system (1.1) is initially prepared so that it is consistent with the appropriately transformed solution to the variational slow system of order $n$ modulo possible errors at $O\left(\varepsilon^{n+1}\right)$, then it remains $O\left(\varepsilon^{n+1}\right)$-close over times of order one.

The remainder of the paper is structured as follows. We next describe a standard non-variational construction of the slow equation. In Section 3, we detail the method of degenerate variational asymptotics and show that the process can be carried out to any order. Section 4 discusses the resulting slow Euler-Lagrange equations. Section 5 contains our main shadowing result, Theorem 5.1. In Section 6, we illustrate the construction by explicitly performing the computation up to second order. The paper concludes with some final remarks.

\section{Nonvariational construction}

In this section, we recall a classical construction of the splitting into slow and fast variables similar to what has been used, for example, by Nershtadt (1984). The result does not depend on the variational structure of the parent system; it only relies on the anti-symmetry of $J$ and the associated oscillatory nature of the fast dynamics.

Theorem 2.1. For $n \geq 0$ fixed, suppose $V \in C^{n+2}$ and set

$$
F_{\mathrm{nv}}^{n}(q)=\sum_{i=0}^{n} f_{i}(q) \varepsilon^{i}
$$

with coefficient functions $f_{i}$ recursively defined via

$$
f_{0}(q)=-J \nabla V(q)
$$

Article submitted to Royal Society 


$$
f_{k}(q)=-J \sum_{i+j=k-1} \mathrm{D} f_{i}(q) f_{j}(q)
$$

For fixed initial positions $q_{0} \in \mathbb{R}^{2 d}$, let $q(t)$ denote a solution to the nonvariational limit system

$$
\dot{q}=F_{\mathrm{nv}}^{n}(q)
$$

with $q(0)=q_{0}$. Let $q_{\varepsilon}(t)$ solve the full parent dynamics (1.1) consistently initialized via $q_{\varepsilon}(0)=q_{0}$ and $p_{\varepsilon}(0)=F_{\mathrm{nv}}^{n}\left(q_{0}\right)$. Then for every $T>0$ there exists $\varepsilon_{0}>0$ and $c=c\left(q_{0}, T\right)$ such that

$$
\sup _{t \in[0, T]}\left\|q_{\varepsilon}(t)-q(t)\right\| \leq c \varepsilon^{n+1}
$$

for all $0<\varepsilon \leq \varepsilon_{0}$.

Proof. Introduce a new fast variable $w_{n+1}=p_{\varepsilon}-F_{\mathrm{nv}}^{n+1}\left(q_{\varepsilon}\right)$, so that

$$
\begin{aligned}
\dot{q}_{\varepsilon} & =F_{\mathrm{nv}}^{n}+w_{n+1}+\varepsilon^{n+1} f_{n+1}, \\
\dot{w}_{n+1} & =\left(\frac{1}{\varepsilon} J-\mathrm{D} F_{\mathrm{nv}}^{n+1}\right) w_{n+1}+\frac{1}{\varepsilon}\left(J F_{\mathrm{nv}}^{n+1}-\nabla V\right)-\mathrm{D} F_{\mathrm{nv}}^{n+1} F_{\mathrm{nv}}^{n+1} .
\end{aligned}
$$

Substituting in the expansion (2.1), we can iteratively determine the $f_{i}$ such that the inhomogeneity in $(2.5 \mathrm{~b})$ is of order $\varepsilon^{n+1}$. This immediately yields $(2.2)$ as a recursive formula for the nonvariational vector field. Left-multiplying $(2.5 \mathrm{~b})$ with $w_{n+1}$ gives

$$
\frac{\mathrm{d}}{\mathrm{d} t}\left\|w_{n+1}\right\| \leq\left\|\mathrm{D} F_{\mathrm{nv}}^{n+1}\right\|\left\|w_{n+1}\right\|+O\left(\varepsilon^{n+1}\right)
$$

so that, if $w_{n+1}=O\left(\varepsilon^{n+1}\right)$ initially, it will remain so for times of order one. As the two last terms in (2.5a) are both $O\left(\varepsilon^{n+1}\right)$, the dynamics is predominantly slow and can be approximated to $O\left(\varepsilon^{n+1}\right)$ by $\dot{q}=F_{\text {nv }}^{n}(q)$ over times of order one.

Remark 1 . It is easy to check that the slow system of Theorem 2.1 is nonvariational with respect to the canonical symplectic structure: the first order term $f_{1}$ in $(2.2)$ is not closed, hence cannot be written as the gradient of a potential. It is more difficult to exclude whether, at least for special $V,(2.3)$ may be variational with respect to a noncanonical structure. Numerical evidence, however, indicates that this is generally not the case. E.g., when $d=1, V$ is a quartic potential, and $\varepsilon>0$ is fixed, solutions of the slow system blow up in a finite time, larger though than the time of validity of the estimate of Theorem 2.1 .

\section{Degenerate variational asymptotics}

We now describe the method of degenerate variational asymptotics. A key ingredient is the construction of a transformation $q_{\varepsilon}=\Phi_{n}[q]$, where $q$ will be the slow variable. We use the square bracket notation to indicate that we seek $\Phi_{n}$ as a functional of $q$ which shall not only depend on $q$ but also on a certain number of its derivatives. We begin by describing the methods in three steps, then we prove that the required transformation can always be found. 
Step 1. Fix $n \in \mathbb{N}$, insert the ansatz

$$
q_{\varepsilon}=\Phi_{n}[q] \equiv \sum_{i=0}^{n} \frac{\varepsilon^{i}}{i !} q^{[i]}
$$

with $q^{[0]}=q$ into the Lagrangian $L_{\varepsilon}\left(q_{\varepsilon}, \dot{q}_{\varepsilon}\right)$, expand, and collect powers of $\varepsilon$. In this process, arbitrary time derivatives which are null-Lagrangians - they do not contribute to the equations of motion - may be added or subtracted as convenient.

Step 2. Choose, iteratively at order $\varepsilon^{1}, \ldots, \varepsilon^{n}$ the coefficients $q^{[1]}, \ldots, q^{[n]}$ as functions of $q$ and its time time derivatives $q^{(1)}, \ldots, q^{(n)}$ in such a way that the expanded Lagrangian is of the form

$$
L_{\varepsilon}\left(q_{\varepsilon}, \dot{q}_{\varepsilon}\right)=\sum_{i=0}^{n} \frac{\varepsilon^{i}}{i !} L^{[i]}(q, \dot{q})+O\left(\varepsilon^{n+1}\right),
$$

where each $L^{[i]}(q, \dot{q})$ is an affine function of $\dot{q}$.

Step 3. Truncate terms of order larger than $\varepsilon^{n}$ and compute the Euler-Lagrange equation of the resulting reduced Lagrangian.

As the notation suggests, we can operate with (3.1) as if it were a power series expansion in $\varepsilon$. In the end, however, this procedure is defining a transformation between $q_{\varepsilon}$, representing the solution in the old, physical coordinates, and $q$, representing the solution in the newly constructed coordinate system.

In Section 6, we illustrate the procedure by explicit computation up to terms of second order. In the following, we shall show that the procedure can be performed to any order. This result is a prerequisite for our main theorem. We write $\alpha$ to denote an $\ell$-tuple of nonnegative integers where, depending on context, $\ell=i$ or $\ell=i-1$ and, following the usual multi-index convention, $\alpha !=\alpha_{1} ! \cdots \alpha_{\ell}$ ! and $|\alpha|=\alpha_{1}+\cdots+\alpha_{\ell}$.

Proposition 3.1. For every fixed $n \in \mathbb{N}$, there exists a transformation of the form (3.1) with coefficients

$$
\frac{q^{[i]}}{i !}=\left(\begin{array}{c}
-\frac{1}{2} \\
i
\end{array}\right) J^{i} q^{(i)}+\sum_{\substack{1 \cdot \alpha_{1}+2 \cdot \alpha_{2}+\cdots \\
+(i-1) \alpha_{i-1} \leq i-1}} T_{\alpha}^{i}(q)\left[\left(q^{(1)}\right)^{\otimes \alpha_{1}}, \ldots,\left(q^{(i-1)}\right)^{\otimes \alpha_{i-1}}\right]
$$

where for $q$ fixed, $T_{\alpha}(q)$ is an $|\alpha|$-linear form in its remaining arguments and $\otimes \alpha$ denotes an $\alpha$-fold repetition of the argument, such that, up to perfect time derivatives,

$$
L_{\varepsilon}\left(q_{\varepsilon}, \dot{q}_{\varepsilon}\right)=L_{\text {slow }}^{n}(q, \dot{q})+\varepsilon^{n+1} L_{R}^{n}\left(q, \dot{q}, \ldots, q^{(n+1)}\right)
$$

with an affine leading contribution

$$
L_{\text {slow }}^{n}(q, \dot{q})=-\frac{1}{2} \dot{q}^{T} R_{n}(q)-V_{n}(q)
$$

containing at most $n$ derivatives of $V$ and a remainder $L_{R}^{n}$ containing at most $n+1$ derivatives of $V$. The perturbation expansions are near-identity in the sense that $\Phi_{n}[q]=q+\varepsilon \Psi_{n}[q], R_{n}(q)=J q+\varepsilon G_{n}(q)$ and $V_{n}(q)=V(q)+\varepsilon W_{n}(q)$. 
Proof. We begin by inserting (3.1) as an ansatz into $L_{\varepsilon}$ and develop into a Taylor series with integral remainder,

$$
L_{\varepsilon}=\sum_{i=0}^{n} \frac{\varepsilon^{i}}{i !} L^{[i]}+\frac{1}{n !} \int_{0}^{\varepsilon}(\varepsilon-s)^{n} L_{s}^{[n+1]} \mathrm{d} s,
$$

where, via the Faà di Bruno formula (Johnson 2002), the $i$ th derivative with respect to $\varepsilon$ of $L_{\varepsilon}$ is given by

$$
\begin{aligned}
\frac{L_{\varepsilon}^{[i]}}{i !}= & \frac{1}{2} \sum_{j+k=i-1}\left(\frac{\dot{q}_{\varepsilon}^{[j]}}{j !}\right)^{T}\left(\frac{\dot{q}_{\varepsilon}^{[k]}}{k !}\right)-\frac{1}{2} \sum_{j+k=i}\left(\frac{\dot{q}_{\varepsilon}^{[j]}}{j !}\right)^{T} J\left(\frac{q_{\varepsilon}^{[k]}}{k !}\right) \\
& -\sum_{1 \cdot \alpha_{1}+\cdots+i \alpha_{i}=i} \frac{1}{\alpha !} D^{|\alpha|} V\left(q_{\varepsilon}\right)\left[\left(\frac{q_{\varepsilon}^{[1]}}{1 !}\right)^{\otimes \alpha_{1}}, \ldots,\left(\frac{q_{\varepsilon}^{[i]}}{i !}\right)^{\otimes \alpha_{i}}\right]
\end{aligned}
$$

and where we set $L^{[i]} \equiv L_{0}^{[i]}$ for every $i \geq 0$.

We will now iteratively construct the $q^{[i]}$ of our transformation such that the corresponding $L_{\varepsilon}^{[i]}$ become affine. At level $i=0$, we set $q^{[0]}=q$. Now let $i \geq 1$ and assume that $q^{[0]}, \ldots, q^{[i-1]}$ have already been constructed. Noting that, for arbitrary $u$ and $v$,

$$
\dot{u}^{T} J v+\dot{v}^{T} J u=2 \dot{u}^{T} J v
$$

up to a perfect derivative, we isolate the two terms which contain the currently undetermined $q^{[i]}$, writing

$$
\begin{aligned}
L^{[i]}= & \frac{i !}{2} \sum_{j+k=i-1}\left(\frac{\dot{q}^{[j]}}{j !}\right)^{T}\left(\frac{\dot{q}^{[k]}}{k !}\right)-\dot{q}^{T} J q^{[i]}-\frac{i !}{2} \sum_{\substack{j+k=i \\
j, k \geq 1}}\left(\frac{\dot{q}^{[j]}}{j !}\right)^{T} J\left(\frac{q^{[k]}}{k !}\right) \\
& -\mathrm{D} V(q) q^{[i]}-\sum_{\substack{1 \cdot \alpha_{1}+\cdots \\
+(i-1) \alpha_{i-1}=i}} \frac{i !}{\alpha !} D^{|\alpha|} V\left(q_{\varepsilon}\right)\left[\left(\frac{q^{[1]}}{1 !}\right)^{\otimes \alpha_{1}}, \ldots,\left(\frac{q^{[i-1]}}{(i-1) !}\right)^{\otimes \alpha_{i-1}}\right] .
\end{aligned}
$$

Let us now explicitly determine the leading order term of the transformation. Substituting in the expression for $q^{[j]}$ for $j=0, \ldots, i-1$ from $(3.3)$, we see that only the two sums on the first line of (3.9) contain the maximal number of $i+1$ time derivatives. Looking only at the leading order contribution, these terms read,

$$
\begin{aligned}
& \left.\frac{i !}{2} \sum_{j+k=i-1}\left(\begin{array}{c}
-\frac{1}{2} \\
j
\end{array}\right)\left(\begin{array}{c}
-\frac{1}{2} \\
k
\end{array}\right)\left(J^{j} \dot{q}^{(j)}\right)^{T} J^{k} \dot{q}^{(k)}-\sum_{\substack{j+k=i \\
j, k \geq 1}}\left(\begin{array}{c}
-\frac{1}{2} \\
j
\end{array}\right)\left(\begin{array}{c}
-\frac{1}{2} \\
k
\end{array}\right)\left(J \dot{q}^{(j)}\right)^{T} J^{k+1} q^{(k)}\right] \\
& \quad=\frac{i !}{2} \dot{q}^{T} J^{i-1} q^{(i)}\left[\sum_{j+k=i-1}\left(\begin{array}{c}
-\frac{1}{2} \\
j
\end{array}\right)\left(\begin{array}{c}
-\frac{1}{2} \\
k
\end{array}\right)+\sum_{j+k=i}\left(\begin{array}{c}
-\frac{1}{2} \\
j
\end{array}\right)\left(\begin{array}{c}
-\frac{1}{2} \\
k
\end{array}\right)-2\left(\begin{array}{c}
-\frac{1}{2} \\
i
\end{array}\right)\left(\begin{array}{c}
-\frac{1}{2} \\
0
\end{array}\right)\right] \\
& \quad=\frac{i !}{2} \dot{q}^{T} J^{i-1} q^{(i)}\left[\left(\begin{array}{c}
-1 \\
i-1
\end{array}\right)+\left(\begin{array}{c}
-1 \\
i
\end{array}\right)-2\left(\begin{array}{c}
-\frac{1}{2} \\
i
\end{array}\right)\right] \\
& =-i ! \dot{q}^{T} J^{i-1} q^{(i)}\left(\begin{array}{c}
-\frac{1}{2} \\
i
\end{array}\right)
\end{aligned}
$$


where the second identity holds modulo perfect derivatives, the third identity is due to the Vandermonde identity and the last identity uses Pascal's rule together with $\left(\begin{array}{l}0 \\ i\end{array}\right)=0$. Since the terms in (3.10) must be annihilated by the term $-\dot{q}^{T} J q^{[i]}$ in equation (3.9), we demand that

$$
q^{[i]}=i !\left(\begin{array}{c}
-\frac{1}{2} \\
i
\end{array}\right) J^{i} q^{(i)}+\text { lower order terms } .
$$

We now proceed to successively eliminate terms which contain $j=i-1, \ldots, 2$ derivatives, until only terms with $j=1$ time derivatives are left. The resulting expressions are combinatorially extremely complex, but the process can be summarized by lumping all terms depending on the same set of derivatives of $q$ into a single multilinear expression. Namely, after full insertion of (3.3) into (3.9), where the $T_{\alpha}^{i}$ are considered ansatz functions which are yet to be determined, elimination of the leading order contribution through (3.11), and the removal of perfect time derivatives, we can write, initially with $j=i-1$ and $W^{i-1} \equiv 0$,

$$
\begin{aligned}
L^{[i]}= & -\left[\dot{q}^{T} J+\mathrm{D} V(q)\right] \sum_{1 \cdot \alpha_{1}+\cdots+j \alpha_{j} \leq j} T_{\alpha}^{i}(q)\left[\left(q^{(1)}\right)^{\otimes \alpha_{1}}, \ldots,\left(q^{(j)}\right)^{\otimes \alpha_{j}}\right] \\
& +\dot{q}^{T} \sum_{1 \cdot \alpha_{1}+\cdots+j \alpha_{j} \leq j} U_{\alpha}^{j}(q)\left[\left(q^{(1)}\right)^{\otimes \alpha_{1}}, \ldots,\left(q^{(j)}\right)^{\otimes \alpha_{j}}\right]+W^{j}(q) \\
= & \dot{q}^{T} \sum_{1 \cdot \alpha_{1}+\cdots+j \alpha_{j}=j}\left(U_{\alpha}^{j}-J T_{\alpha}^{i}\right)(q)\left[\left(q^{(1)}\right)^{\otimes \alpha_{1}}, \ldots,\left(q^{(j)}\right)^{\otimes \alpha_{j}}\right] \\
& -\mathrm{D} V(q) \sum_{1 \cdot \alpha_{1}+\cdots+j \alpha_{j} \leq j} T_{\alpha}^{i}(q)\left[\left(q^{(1)}\right)^{\otimes \alpha_{1}}, \ldots,\left(q^{(j)}\right)^{\otimes \alpha_{j}}\right] \\
& +\dot{q}^{T} \sum_{1 \cdot \alpha_{1}+\cdots}\left(U_{\alpha}^{j}-J T_{\alpha}^{i}\right)(q)\left[\left(q^{(1)}\right)^{\otimes \alpha_{1}}, \ldots,\left(q^{(j-1)}\right)^{\otimes \alpha_{j-1}}\right] \\
& +W^{j}(q) .
\end{aligned}
$$

The first sum on the right of (3.12) contains all terms with the maximal $j+1$ time derivatives which can be eliminated by setting $T_{\alpha}^{i} \equiv-J U_{\alpha}^{j}$ for the indices which appear in the first sum. This choice must be substituted back into the second sum on the right of (3.12). After possible integration by parts, i.e. the removal of perfect derivatives, these terms can be merged into the third sum on the right of (3.12) if they contain at least one time derivative or into $W^{j}$ if they do not. Rearranging terms, we again find an expression of the form (3.12) with $j$ decreased by 1 . This procedure can be repeated until $j=1$ when the remaining expression is affine and all the $T_{\alpha}^{i}$ which appear in (3.3) are fully determined. This completes the construction of the transformation (3.1).

To see that the remainder Lagrangian has the indicated form, it suffices to insert the transformation into the Taylor remainder from (3.6), noting that the highest possible time derivative in the resulting expression is of order $n+1$.

Remark 2. Proposition 3.1 and all subsequent results in this paper remain valid and otherwise unchanged if we add arbitrary point transformations, i.e. functions of $q$ only, to the $q^{[i]}$ at each order $i \geq 1$. 
Remark 3. In Proposition 3.1 we are able to state a general expression for the leading term of the transformation at any order. A similarly explicit expression for all terms, however, appears to be combinatorially too complex to be useful.

\section{Euler-Lagrange equations}

Generally, if $L(q, \dot{q}, \ldots)$ is a Lagrangian, the corresponding Euler-Lagrange equation is equivalent to computing critical points of the action

$$
S=\int_{t_{1}}^{t_{2}} L(q, \dot{q}, \ldots) \mathrm{d} t
$$

with respect to variations $\delta q$ that vanish at $t_{1}$ and $t_{2}$. For the slow Lagrangian $L_{\text {slow }}^{n}$ from Proposition 3.1, we obtain after integration by parts that

$$
\delta S_{\text {slow }}^{n}=\int_{t_{1}}^{t_{2}} \delta q^{T}\left(\frac{1}{2}\left(\mathrm{D} R_{n}(q)-\mathrm{D} R_{n}(q)^{T}\right) \dot{q}-\nabla V_{n}(q)\right) \mathrm{d} t
$$

Thus, setting

$$
J_{n}(q)=\frac{\mathrm{D} R_{n}(q)-\mathrm{D} R_{n}(q)^{T}}{2}
$$

we can write the slow Euler-Lagrange equation as

$$
\mathrm{EL}_{\text {slow }}^{n}[q] \equiv-J_{n}(q) \dot{q}+\nabla V_{n}(q)=0 \text {. }
$$

In the following, if $f_{\varepsilon}:[0, T] \rightarrow \mathbb{R}^{2 d}$ denotes a family of functions defined for $\varepsilon \in\left(0, \varepsilon_{0}\right]$, we say that $f_{\varepsilon}=\mathcal{O}\left(\varepsilon^{m}\right)$ if $\mathrm{d}^{j} / \mathrm{d} t^{j} f_{\varepsilon}=O\left(\varepsilon^{m}\right)$ as $\varepsilon \rightarrow 0$ for every $j \in \mathbb{N}_{0}$. As a first basic result, we obtain that solutions to (4.4) are slow in this sense. For simplicity, we assume that $V$ is smooth throughout.

Proposition 4.1. Let $q$ denote a family of solutions to the slow Euler-Lagrange equation (4.4), implicitly parametrized by $\varepsilon$, with $q(0)$ fixed. Then $q=\mathcal{O}(1)$.

Proof. Recall that $J_{n}(q)=J q+\varepsilon G_{n}(q)$. Thus, on every fixed bounded set, $J_{n}(q)$ is uniformly invertible for $\varepsilon$ small enough. Thus, for any fixed $T>0$, uniform boundedness of $\sup _{t \in[0, T]}|q(t)|$ for small $\varepsilon$ follows by a direct application of the Gronwall lemma to (4.4). Further, uniform boundedness of $\dot{q}$ follows directly from the slow equation of motion; boundedness of higher time derivatives is similarly obtained by successive time differentiation of (4.4) followed by expressing the time derivatives as functions of $q$ upon resubstitution of $\dot{q}$ from the slow equation, which can always be done as $J_{n}(q)$ is uniformly invertible.

Remark 4. When $V$ is convex, the restriction on $\varepsilon$ in the proof of Proposition 4.1 can be chosen uniform in $T$. This can be seen as follows. For fixed $q(0)$, choose $b>|V(q(0))|$. Convexity of $V$ implies that there exists $B$ such that $|z|<B$ whenever $|V(z)|<b$. Recall that $V_{n}(q)=V(q)+\varepsilon W_{n}(q)$. Continuity of $W_{n}$ implies that there exists $c$ such that $\left|W_{n}(z)\right|<c$ for all $|z|<B$. Choose $\varepsilon<|b-V(q(0))| /(2 c)$. Since $V_{n}$ is conserved under the evolution of (4.4), this means that $V(q)$ is constrained to a ball of radius $|b-V(q(0))| / 2$ about $V_{n}$ so that, in particular, $|V(q)|<b$ and $q$ remains correspondingly constrained to $|q|<B$ for all times. 
Next, we estimate the residue when inserting the transformed solution of the slow Euler-Lagrange equation into the expression for the full Euler-Lagrange equation.

Proposition 4.2. As before, let $q$ denote an $\varepsilon$-parametrized family of solutions to the slow Euler-Lagrange equation (4.4) with $q(0)$ fixed. Then $\operatorname{EL}_{\varepsilon}\left[\Phi_{n}[q]\right]=\mathcal{O}\left(\varepsilon^{n+1}\right)$.

In the proof, we make use of a simple lemma.

Lemma 4.3. Let

$$
\mathcal{A}_{\varepsilon}=I+\varepsilon \sum_{i=0}^{k} a_{\varepsilon}^{i}(t) \frac{\mathrm{d}^{i}}{\mathrm{~d} t^{i}}
$$

be a family of linear operators with any finite number of nonzero coefficients $a_{\varepsilon}^{i}=$ $\mathcal{O}(1)$. Suppose further that $f_{\varepsilon}$ is a family of $\mathcal{O}(1)$-functions, $m$ times differentiable in $\varepsilon$ for some $m \in \mathbb{N}_{0}$. Then $\mathcal{A}_{\varepsilon} f_{\varepsilon}=\mathcal{O}\left(\varepsilon^{m}\right)$ implies $f_{\varepsilon}=\mathcal{O}\left(\varepsilon^{m}\right)$.

Proof. Expand $f_{\varepsilon}$ into a Taylor series of order $m$ and match coefficients order by order.

Proof of Proposition 4.2. We begin by computing the full Euler-Lagrange equation in the transformed frame of reference. Varying the corresponding action, we find

$$
\begin{aligned}
\delta S & =\delta \int_{t_{1}}^{t_{2}} L_{\varepsilon}\left(\Phi_{n}[q], \frac{\mathrm{d}}{\mathrm{d} t} \Phi_{n}[q]\right) \mathrm{d} t \\
& =\int_{t_{1}}^{t_{2}} \delta q^{T} \mathrm{D} \Phi_{n}[q]^{*}\left(\mathrm{D}_{q} L_{\varepsilon}\left(\Phi_{n}[q], \frac{\mathrm{d}}{\mathrm{d} t} \Phi_{n}[q]\right)-\frac{\mathrm{d}}{\mathrm{d} t} \mathrm{D}_{\dot{q}} L_{\varepsilon}\left(\Phi_{n}[q], \frac{\mathrm{d}}{\mathrm{d} t} \Phi_{n}[q]\right)\right) \mathrm{d} t \\
& =\int_{t_{1}}^{t_{2}} \delta q^{T} \mathrm{D}_{n}[q]^{*} \mathrm{EL}_{\varepsilon}\left[\Phi_{n}[q]\right] \mathrm{d} t
\end{aligned}
$$

where $\mathrm{D} \Phi_{n}^{*}$ denotes the adjoint of $\mathrm{D} \Phi_{n}$. Since, by Proposition 3.1,

$$
L_{\text {slow }}^{n}(q, \dot{q})=L_{\varepsilon}\left(\Phi_{n}(q), \frac{\mathrm{d}}{\mathrm{d} t} \Phi_{n}(q)\right)-\varepsilon^{n+1} L_{R}^{n}\left(q, \ldots, q^{(n+1)}\right),
$$

we correspondingly have that

$$
\mathrm{EL}_{\text {slow }}^{n}[q]=\mathrm{D}_{n}[q]^{*} \mathrm{EL}_{\varepsilon}\left[\Phi_{n}[q]\right]-\varepsilon^{n+1} \mathrm{EL}_{R}^{n}[q],
$$

where the first term on the right was derived in (4.6) and the second term on the right denotes the Euler-Lagrange equation of the remainder which, without further computation, is seen to be some expression with smooth dependence on at most $2(n+1)$ derivatives of $q$. Thus, supposing $q$ solves the slow Euler-Lagrange equation, $\mathrm{EL}_{R}^{n}[q]=\mathcal{O}(1)$ and, moreover, the left hand expression in (4.8) is zero, so that

$$
\mathrm{D}_{n}[q]^{*} \mathrm{EL}_{\varepsilon}\left[\Phi_{n}[q]\right]=\mathcal{O}\left(\varepsilon^{n+1}\right) .
$$

Finally, recalling that $\Phi_{n}$ is a near-identity transformation, we observe that $\operatorname{D} \Phi_{n}[q]^{*}$ is of form (4.5). The claim thus follows by Lemma 4.3. 


\section{Shadowing theorem}

We are now in a position to state and prove our main shadowing result. The proof is in essence an application of the nonvariational splitting introduced in Section 2 to the equation satisfied by the difference between the full motion and the slow motion.

Theorem 5.1. Suppose that $V$ is smooth. For $n \geq 0$ and $q_{0} \in \mathbb{R}^{2 d}$ fixed, let $q$ denote a solution of the slow Euler-Lagrange equation (4.4) with $q(0)=q_{0}$. Let $q_{\varepsilon}$ solve the full parent dynamics (1.1) consistently initialized via $q_{\varepsilon}(0)=\left.\Phi_{n}[q]\right|_{t=0}$ and $p_{\varepsilon}(0)=\left.\frac{\mathrm{d}}{\mathrm{d} t} \Phi_{n}[q]\right|_{t=0}$. Then for every fixed $T>0$ there exist $\varepsilon_{0}>0$ and $c=c\left(q_{0}, T\right)$ such that

$$
\sup _{t \in[0, T]}\left\|q_{\varepsilon}(t)-\Phi_{n}[q(t)]\right\| \leq c \varepsilon^{n+1}
$$

for all $0<\varepsilon \leq \varepsilon_{0}$

Proof. We set $z=\Phi_{n}[q]$. By Proposition 4.2, $\mathrm{EL}_{\varepsilon}[z]=\mathcal{O}\left(\varepsilon^{n+1}\right)$. The equivalence of the model equation (1.1) and its Euler-Lagrange equation (1.3) allows us to write

$$
\begin{aligned}
\dot{z} & =r \\
\varepsilon \dot{r} & =J r-\nabla V(z)+\mathcal{O}\left(\varepsilon^{n+1}\right) .
\end{aligned}
$$

We now let $u=q_{\varepsilon}-z, v=\dot{u}$, and-following the construction in the proof of Theorem 2.1 -introduce the fast variable $w=v-F^{n+1}(z, u)$ with $F^{n+1}$ yet to be determined. Then

$$
\begin{aligned}
\dot{u}= & w+F^{n+1} \\
\dot{w}= & \frac{1}{\varepsilon}\left(J v+\nabla V(z)-\nabla V\left(q_{\varepsilon}\right)\right)-\mathrm{D}_{z} F^{n+1} \dot{z}-\mathrm{D}_{u} F^{n+1} \dot{u}+\mathcal{O}\left(\varepsilon^{n}\right) \\
= & \left(\frac{1}{\varepsilon} J-\mathrm{D}_{u} F^{n+1}\right) w+\frac{1}{\varepsilon}\left(J F^{n+1}+\int_{0}^{1} \mathrm{D} \nabla V(z-\lambda u) u \mathrm{~d} \lambda\right) \\
& -\mathrm{D}_{z} F^{n+1} \dot{z}-\mathrm{D}_{u} F^{n+1} F^{n+1}+\mathcal{O}\left(\varepsilon^{n}\right) .
\end{aligned}
$$

Expanding

$$
F^{n+1}(z, u)=\sum_{i=0}^{n+1} f_{i}(u, z) \varepsilon^{i},
$$

we can, as in the proof of Theorem 2.1, choose the $f_{i}$ order by order as to eliminate all but the first term on the right of (5.3b) up to a remainder of $O\left(\varepsilon^{n+1}\right)$. The Gronwall lemma yields, once again, that if $w=O\left(\varepsilon^{n+1}\right)$ initially, it will remain so for times of order one. (Note that $w$ is only $O\left(\varepsilon^{n+1}\right)$ but not $\mathcal{O}\left(\varepsilon^{n+1}\right)$ as it contains small amplitude fast oscillations with frequencies of order $\varepsilon^{-1}$.)

Hence, $u$ satisfies

$$
\dot{u}=F^{n+1}(u)+O\left(\varepsilon^{n+1}\right) .
$$

When $q_{\varepsilon}$ is consistently initialized as assumed, $u=O\left(\varepsilon^{n+1}\right)$ initially, so it remains so for times of order one. This directly implies the claim of the theorem. 
Remark 5. As the coefficients of a Taylor series are uniquely defined, a variation of the proof of Theorem 5.1 can be used to show that any parent system which is an $O\left(\varepsilon^{n+1}\right)$ perturbation of (1.1) must share the same nonvariational slow system $\dot{q}=F_{\text {nv }}^{n}(q)$. In particular, the nonvariational slow vectorfield $F_{\mathrm{nv}}^{n}$ and the variational slow vectorfield $F_{\text {var }}^{n}=J_{n}^{-1}(q) \nabla V_{n}(q)$ arising from (4.4) differ only by $O\left(\varepsilon^{n+1}\right)$.

Remark 6. Using the explicit expressions for the first and second order model we derive in Section 6 below, it is easy to simulate the model problem numerically. Such experiments suggest that in general we cannot expect an order of accuracy of the shadowing result, or a time scale of validity better than what is provided by Theorem 5.1. However, it has been observed by Oliver (2006) that in the very special situation of first order slow equation for a harmonic potential, the order of accuracy is actually better by one as compared to the generic case.

Remark 7. The proof of Theorem 5.1 requires only that $V \in C^{2(n+1)}$. Although we can then only assert that the right hand side in (4.9) is $O\left(\varepsilon^{n+1}\right)$, careful tracking of time differentiability shows that all other arguments will continue to hold true. However, it is not clear whether this restriction is really necessary as the slow Euler-Lagrange equation at order $\varepsilon^{i}$ depends on at most $i+1$ derivatives of $V$.

\section{Explicit expressions}

In this section, we derive the variational slow equation and the associated change of coordinates up to second order. The expressions we write out are unambiguous by noting that all vectors are column vectors, the symbol $\mathrm{D}$ acts as a row vector, $\nabla$ as a column vector, Hess $=\mathrm{D} \nabla$ denotes the Hessian, and $\Delta$ the Laplace operator.

Step 1 . Inserting the ansatz $q_{\varepsilon}=q+\varepsilon q^{\prime}+\frac{1}{2} \varepsilon^{2} q^{\prime \prime}$ for the second order transformation into $L_{\varepsilon}\left(q_{\varepsilon}, \dot{q}_{\varepsilon}\right)$ and expanding in powers of $\varepsilon$, we obtain, up to terms of order $\varepsilon^{3}$ and perfect time derivatives, that

$$
L_{\varepsilon}=L^{[0]}+\varepsilon L^{[1]}+\frac{1}{2} \varepsilon^{2} L^{[2]}
$$

with

$$
\begin{aligned}
& L^{[0]}=-\frac{1}{2} \dot{q}^{T} J q-V(q), \\
& L^{[1]}=\frac{1}{2}|\dot{q}|^{2}-\mathrm{D} V(q) q^{\prime}-\dot{q}^{T} J q^{\prime}, \\
& L^{[2]}=2 \dot{q}^{T} \dot{q}^{\prime}-q^{T} \mathrm{D} \nabla V(q) q^{\prime}-\mathrm{D} V(q) q^{\prime \prime}-\dot{q}^{T} J q^{\prime}-\dot{q}^{T} J q^{\prime \prime} .
\end{aligned}
$$

Step 2. The leading order Lagrangian is already affine. This is generally necessary for the construction to work. At first order, we need to remove the quadratic term $\frac{1}{2}|\dot{q}|^{2}$ from $L^{[1]}$. This requirement implies

$$
q^{\prime}=-\frac{1}{2} J \dot{q}+q_{\text {point }}^{\prime},
$$

where $q_{\text {point }}^{\prime}$ is an arbitrary function of $q$. For reasons that will become apparent later, we consider choices which are proportional to the first term under the substitution of the time derivative by the leading order slow equation. This singles out the family of transformations

$$
q^{\prime}=-\frac{1}{2} J \dot{q}+\mu \nabla V(q)
$$


where $\mu \in \mathbb{R}$ remains arbitrary. Substituting this expression back into (6.1b) we find that the first order Lagrangian is of the form

$$
L^{[1]}=-\frac{1}{2} \dot{q}^{T} R_{1}(q)-V_{1}(q)
$$

with

$$
R_{1}=(1+2 \mu) J \nabla V \quad \text { and } \quad V_{1}=\mu|\nabla V|^{2} .
$$

Moving to the next order, we correspondingly obtain

$$
\begin{aligned}
L^{[2]}= & -\frac{3}{4} \dot{q}^{T} J \ddot{q}+\frac{3}{2} \mu \dot{q}^{T} \mathrm{D} \nabla V \dot{q}+\frac{1}{4} \dot{q}^{T} J \mathrm{D} \nabla V J \dot{q}+\frac{1}{2} \mu \ddot{q}^{T} \nabla V+\mu \nabla V^{T} \mathrm{D} \nabla V J \dot{q} \\
& -\mu^{2} \nabla V^{T} \mathrm{D} \nabla V \nabla V-\mu^{2} \dot{q}^{T} \mathrm{D} \nabla V J \nabla V-\mathrm{D} V q^{\prime \prime}-\dot{q}^{T} J q^{\prime \prime} .
\end{aligned}
$$

To render this expression affine, we must choose

$$
q^{\prime \prime}=-\frac{3}{4} \ddot{q}+\frac{1}{4} \mathrm{D} \nabla V J \dot{q}-\left(\mu-\frac{3}{4}\right) J \mathrm{D} \nabla V \dot{q}+q_{\text {point }}^{\prime \prime}
$$

where, arguing as before, we choose as free point transformation the three-parameter family of vector fields

$$
q_{\text {point }}^{\prime \prime}=\nu_{1} \nabla V \Delta V+\nu_{2} \mathrm{D} \nabla V \nabla V+\nu_{3} J \mathrm{D} \nabla V J \nabla V
$$

Inserting this choice back into $L^{[2]}$, the second order Lagrangian reads

$$
L^{[2]}=-\frac{1}{2} \dot{q}^{T} R_{2}(q)-V_{2}
$$

with

$$
R_{2}(q)=-\left(\frac{1}{4}-\mu-\nu_{2}\right) J \mathrm{D} \nabla V \nabla V-\left(\frac{3}{4}+\nu_{3}-\mu-\mu^{2}\right) \mathrm{D} \nabla V J \nabla V+\nu_{1} J \nabla V \Delta V
$$

and

$$
V_{2}=\frac{1}{2}\left(\mu^{2}+\nu_{2}+\nu_{3}\right) \nabla V^{T} \mathrm{D} \nabla V \nabla V+\frac{1}{2}\left(\nu_{1}-\nu_{3}\right)|\nabla V|^{2} \Delta V .
$$

Step 3. According to (4.4), the general Euler-Lagrange equation is of the form

$$
\left(J+\varepsilon J_{1}+\frac{1}{2} \varepsilon^{2} J_{2}\right) \dot{q}=\nabla\left(V+\varepsilon V_{1}+\frac{1}{2} \varepsilon^{2} V_{2}\right)
$$

where $V_{1}, V_{2}$ are given above and $J_{1}, J_{2}$ can be computed via (4.3). In particular, under the assumption that $d=1$ which permits a number of algebraic simplifications, we find that

$$
J_{1}=\left(\frac{1}{2}+\mu\right) \Delta V J
$$

and

$$
\begin{gathered}
J_{2}=\frac{1}{2}\left[\left(\mu+\nu_{1}+\nu_{2}-\frac{1}{4}\right) \operatorname{D} V \nabla \Delta V+\left(\mu+\nu_{2}-\frac{1}{4}\right)|\operatorname{Hess} V|^{2}\right. \\
\left.+2\left(\mu^{2}+\mu-\nu_{3}-\frac{3}{4}\right) \operatorname{det} \operatorname{Hess} V+\nu_{1}(\Delta V)^{2}\right] J
\end{gathered}
$$


We conclude this section with two interesting observations. First, the transformation can be chosen such that $J_{i}=0$ for $i \geq 1$ so that the resulting symplectic structure is canonical. By inspection, this is the case when

$$
\mu=-\frac{1}{2}, \quad \nu_{1}=0, \quad \nu_{2}=\frac{3}{4}, \quad \text { and } \quad \nu_{3}=1 .
$$

Second, the transformation can always be chosen such that the transformation, when applied to a solution of the resulting slow Euler-Lagrange equation, is of the form $q_{\varepsilon}=q+\mathcal{O}\left(\varepsilon^{n+1}\right)$. We speak of a near-near identity transformation as it can be replaced by the exact identity in the statement of Theorem 5.1 without reducing the order of accuracy. To see how this case arises, first note that the Euler-Lagrange equation computed up to $O(\varepsilon)$ reads

$$
\left(1+\varepsilon\left(\frac{1}{2}+\mu\right) \Delta V\right) J \dot{q}=\nabla V+2 \varepsilon \mu \mathrm{D} \nabla V \nabla V .
$$

Using this expression to consistently eliminate all time derivatives from the second order transformation $\Phi_{2}$, we find that

$$
\begin{aligned}
q_{\varepsilon}= & q+\varepsilon\left(\mu-\frac{1}{2}\right) \nabla V+\frac{1}{2} \varepsilon^{2}\left[\left(\frac{1}{2}+\mu+\nu_{1}\right) \nabla V \Delta V\right. \\
& \left.+\left(\nu_{2}-2 \mu+\frac{1}{4}\right) \mathrm{D} \nabla V \nabla V+\left(\mu+\nu_{3}-\frac{3}{2}\right) J \mathrm{D} \nabla V J \nabla V\right]+\mathcal{O}\left(\varepsilon^{3}\right) .
\end{aligned}
$$

Hence, all terms except for the $\mathcal{O}\left(\varepsilon^{3}\right)$ remainder vanish if we choose

$$
\mu=\frac{1}{2}, \quad \nu_{1}=-1, \quad \nu_{2}=\frac{3}{4}, \quad \text { and } \quad \nu_{3}=1 .
$$

Theorem 5.1 assures that the order of the approximation is not affected by the values of the free parameters. Each choice of parameters and indeed each choice of $q_{\text {point }}^{\prime}$ and $q_{\text {point }}^{\prime \prime}$ leads to asymptotically equivalent slow equations.

\section{Discussion}

The present paper gives a complete account of the approximation properties of the slow limit equation derived via degenerate variational asymptotics to any finite order $n$ in a simple, finite dimensional model setting. There are various obvious generalizations of our results as well as harder questions which are largely open.

First, as already seen in a special case in Section 6, there is always the possibility of choosing a "near-near identity" transformation in the following way. Starting at order $i=1$, set $q_{\text {point }}^{[i]}=-q^{[i]}$, use the slow Euler-Lagrange equation of order $i-1$ to eliminate all time derivatives from $q_{\text {point }}^{[i]}$, subtract any carry-overs from the previous iteration level, and compute the Euler-Lagrange equation to order $i$. Using this $O\left(\varepsilon^{i}\right)$ equation, recompute all $q_{\text {point }}^{[j]}$ for $j=1, \ldots, i$, carrying all terms up to order $i$. Then iterate up to order $i+1$. The resulting transformation will be zero up to slow terms of $O\left(\varepsilon^{i+1}\right)$, yet satisfy the requirements of splitting the Lagrangian as in Proposition 3.1.

Second, the construction obviously extends to general nondegenerate mass matrices and to position dependent gyroscopic terms. We note that the construction in Proposition 3.1 can always be performed so long as $J(q)$ is invertible. If $J$ is singular, there is generally slow motion along the singular directions, known as guiding center motion (Littlejohn 1983); it is open whether this can be analyzed in our framework. 
Third, numerical evidence and results at low order by Gottwald et al. (2007) suggest that it is possible to trade approximation order for time scale of validity, i.e., that the slow equation shadow the full system to $O\left(\varepsilon^{n+1-j}\right)$ on time scales $O\left(\varepsilon^{-j}\right)$ provided the potential $V$ is convex. A general proof is still open.

Fourth, state of the art Hamiltonian normal form theory yields exponential estimates which are based on an optimal truncation of a perturbation series for the Hamiltonian. In this framework, Nehorošev (1977) type estimates can be used to prove exponential estimates for the tracking of the slow manifold as a phase space object, see also Benettin et al. 1987, Gelfreich \& Lerman (2002), MacKay (2004) and Uldall Kristiansen \& Wulff (2012). Cotter \& Reich (2006), in particular, obtain specific results for our model (1.1), and Wirosoetisno (2004) and Vanneste (2013) discuss closely related model problems. We believe that exponential estimates should be possible within our framework as well, but they are considerably more difficult due to the interactions between the simultaneous asymptotic series for the symplectic structure matrix and the Hamiltonian.

Finally, we believe that the methods are not only applicable, but particularly useful for problems in partial differential equations where the freedom to add arbitrary point transformations at each order can be used to control the functional setting of the resulting limit equations (Oliver \& Vasylkevych 2013, 2014). This is difficult in the classical setting of Hamiltonian perturbation theory where transformations with frozen structure operator typically lead to buildup of unbounded operators in the perturbation series.

We thank Bin Cheng, Colin Cotter, Holger Dullin, Sebastian Reich, Jacques Vanneste, and Sergiy Vasylkevych for stimulating discussions, and gratefully acknowledge hospitality and support through the Research-in-Pairs program of the Mathematisches Forschungsinstitut Oberwolfach where this work was begun. The work of GAG was further supported by the Australian Research Council, the work of MO by German Science Foundation grant OL-155/3.

\section{References}

Benettin, G., Galgani, L. \& Giorgilli, A. 1987 Realization of holonomic constraints and freezing of high frequency degrees of freedom in the light of classical perturbation theory I. Comm. Math. Phys. 113, 87-103.

Cheng, B. \& Mahalov, A. 2013 Time-averages of fast oscillatory systems. Discrete Contin. Dyn. Syst. Ser. S 6, 1151-1162.

Cotter, C. J. \& Reich, S. 2006 Semigeostrophic particle motion and exponentially accurate normal forms. Multiscale Model. Simul. 5, 476-496.

Cullen, M. J. P. 2006 A Mathematical Theory of Large-Scale Atmosphere/Ocean Flow. London: Imperial College Press.

Frank, J. E. \& Gottwald, G. A. 2013 Stochastic homogenization for an energy conserving multi-scale toy model of the atmosphere. Phys. D 254, 46-56.

Gelfreich, V. \& Lerman, L. 2002 Almost invariant elliptic manifold in a singularly perturbed Hamiltonian system. Nonlinearity 15, 447-457.

Gottwald, G. A., Oliver, M. \& Tecu, N. 2007 Long-time accuracy for approximate slow manifolds in a finite-dimensional model of balance. J. Nonlinear Sci. 17, 283-307.

Johnson, W. P. 2002 The curious history of Faà di Bruno's formula. Amer. Math. Monthly 109, 217-234. 
Littlejohn, R. G. 1983 Variational principles of guiding centre motion. J. Plasma Phys. 29, 111-125.

MacKay, R. S. 2004 Slow manifolds. In Energy Localisation and Transfer (ed. T. Dauxois, A. Litvak-Hinenzon, R. MacKay \& A. Spanoudaki). Singapore: World Scientific, pp. 149-192.

Murdock, J. 2003 Normal forms and unfoldings for local dynamical systems. New York: Springer-Verlag.

Neĭshtadt, A. I. 1984 The separation of motions in systems with rapidly rotating phase. $J$. Appl. Math. Mech. 48 (1985), 133-139. Translated from Prikl. Mat. Mekh. 48, 197-204.

Nehorošev, N. N. 1977 An exponential estimate of the time of stability of nearly integrable Hamiltonian systems. Russ. Math. Surv. 32, 1-65. Translated from Uspehi Mat. Nauk 32, 5-66, 287.

Oliver, M. 2006 Variational asymptotics for rotating shallow water near geostrophy: a transformational approach. J. Fluid Mech. 551, 197-234.

Oliver, M. \& Vasylkevych, S. 2011 Hamiltonian formalism for models of rotating shallow water in semigeostrophic scaling. Discret. Contin. Dyn. S. 31, 827-846.

Oliver, M. \& Vasylkevych, S. 2013 Generalized LSG models with spatially varying Coriolis parameter. Geophys. Astrophys. Fluid Dyn. 107, 259-276.

Oliver, M. \& Vasylkevych, S. 2014 A new construction of modified equations for variational integrators. Submitted for publication.

Popescu, M. 2009 Totally singular Lagrangians and affine Hamiltonians. Balkan J. Geom. Appl. 14, 60-71.

Salmon, R. 1985 New equations for nearly geostrophic flow. J. Fluid Mech. 153, 461-477.

Salmon, R. 1998 Lectures on Geophysical Fluid Dynamics. Oxford: Oxford University Press.

Sanders, J., Verhulst, F. \& Murdock, J. 2007 Averaging Methods in Nonlinear Dynamical Systems. New York: Springer-Verlag.

Uldall Kristiansen, K. \& Wulff, C. 2012 Exponential estimates of slow manifolds. Preprint, arXiv: $1208.4219 \mathrm{v} 1$.

Vanneste, J. 2013 Balance and spontaneous wave generation in geophysical flows. Annu. Rev. Fluid Mech. 45, 147-172.

Wirosoetisno, D. 2004 Exponentially accurate balance dynamics. Adv. Diff. Eq. 9, 177196. 